\title{
On the reproducibility of science: Unique Identification of Research Resources in the Biomedical Literature
}

Scientific reproducibility has been at the forefront of many news stories and there exist numerous initiatives to help address this problem. We pose that a contributor is simply a lack of specificity that is required to enable adequate research reproducibility. In particular, the inability to uniquely identify research resources, such as antibodies and model organisms, makes it difficult or impossible to reproduce experiments even where the science is otherwise sound. In order to better understand the magnitude of this problem, we designed an experiment to ascertain the "identifiability" of research resources in the biomedical literature. We evaluated recent journal articles in the fields of Neuroscience, Developmental Biology, Immunology, Cell and Molecular Biology and General Biology, selected randomly based on a diversity of impact factors for the journals, publishers, and experimental method reporting guidelines. We attempted to uniquely identify model organisms (mouse, rat, zebrafish, worm, fly and yeast), antibodies, knockdown reagents (morpholinos or RNAi), constructs, and cell lines. Specific criteria were developed to determine if a resource was uniquely identifiable, and included examining relevant repositories (such as model organism databases, and the Antibody Registry), as well as vendor sites. The results of this experiment show that $54 \%$ of resources are not uniquely identifiable in publications, regardless of domain, journal impact factor, or reporting requirements. For example, in many cases the organism strain in which the experiment was performed or antibody that was used could not be identified. Our results show that identifiability is a serious problem for reproducibility. Based on these results, we provide recommendations to authors, reviewers, journal editors, vendors, and publishers. Scientific efficiency and reproducibility depend upon a research-wide improvement of this substantial problem in science today. 


\section{Authors:}

2 Nicole A. Vasilevsky ${ }^{1 \S}$, Matthew H. Brush ${ }^{1}$, Holly Paddock² ${ }^{2}$ Laura Ponting ${ }^{3}$, Shreejoy J.

3 Tripathy ${ }^{4}$, Gregory M. LaRocca ${ }^{4}$, Melissa A. Haendel ${ }^{1}$

$4 \quad{ }^{1}$ Ontology Development Group, Library, Oregon Health \& Science University, Portland, OR, 5 USA

$6 \quad{ }^{2}$ Zebrafish Information Framework, University of Oregon, Eugene, OR, USA

$7 \quad{ }^{3}$ FlyBase, Department of Genetics, University of Cambridge, Cambridge, UK

$8{ }^{4}$ Department of Biological Sciences and Center for the Neural Basis of Cognition, Carnegie

9 Mellon University, Pittsburgh, Pennsylvania, USA

10 §Corresponding author

11 Nicole Vasilevsky

12 OHSU, LIB

133181 SW Sam Jackson Park Road

14 Portland, OR 97239

15 503-806-6900

16 vasilevs@ohsu.edu 


\section{Abstract}

18 Scientific reproducibility has been at the forefront of many news stories and there exist 19 numerous initiatives to help address this problem. We pose that a contributor is simply a lack of

20 specificity that is required to enable adequate research reproducibility. In particular, the inability

21 to uniquely identify research resources, such as antibodies and model organisms, makes it

22 difficult or impossible to reproduce experiments even where the science is otherwise sound. In

23 order to better understand the magnitude of this problem, we designed an experiment to ascertain

24 the "identifiability" of research resources in the biomedical literature. We evaluated recent journal

25 articles in the fields of Neuroscience, Developmental Biology, Immunology, Cell and Molecular

26 Biology and General Biology, selected randomly based on a diversity of impact factors for the

27 journals, publishers, and experimental method reporting guidelines. We attempted to uniquely

28 identify model organisms (mouse, rat, zebrafish, worm, fly and yeast), antibodies, knockdown

29 reagents (morpholinos or RNAi), constructs, and cell lines. Specific criteria were developed to

30 determine if a resource was uniquely identifiable, and included examining relevant repositories

31 (such as model organism databases, and the Antibody Registry), as well as vendor sites. The

32 results of this experiment show that $54 \%$ of resources are not uniquely identifiable in

33

34

35

36

37

38

publications, regardless of domain, journal impact factor, or reporting requirements. For example, in many cases the organism strain in which the experiment was performed or antibody that was used could not be identified. Our results show that identifiability is a serious problem for reproducibility. Based on these results, we provide recommendations to authors, reviewers, journal editors, vendors, and publishers. Scientific efficiency and reproducibility depend upon a research-wide improvement of this substantial problem in science today.

39

40

41

42

43

44

45

46

47

48

49

50

51

52

53

54

55

56

57

58

59

60

61

62

63

64

\section{Introduction}

The scientific method relies on the ability of scientists to reproduce and build upon each other's published results. Although it follows that the prevailing publication model should support this objective, it is becoming increasingly apparent that it falls short (Haendel, Vasilevsky, and Wirz 2012; de Waard 2010). This failure was highlighted in a recent Nature report from researchers at the Amgen corporation, who found that only $11 \%$ of the academic research in the literature was reproducible by their groups (Begley and Ellis 2012). Further alarm is raised by the fact that retraction rates, due in large part to a lack of reproducibility, have steadily increased since the first paper was retracted in 1977 (Cokol, Ozbay, and Rodriguez-Esteban 2008). While many factors are likely at play here, perhaps the most basic requirement for reproducibility holds that the materials reported in a study can be uniquely identified and obtained, such that experiments can be reproduced as faithfully as possible. Here, we refer to reproducibility defined as the "conditions where test results are obtained with the same method on identical test materials in different laboratories with different operators using different equipment” (ISO 5725-1:1994 1994). This information is meant to be documented in the 'materials and methods' of journal articles, but as many can attest, the information provided there is often not adequate for this task. Such a fundamental shortcoming costs time and resources, and prevents efficient turns of the research cycle whereby research findings are validated and extended toward new discoveries. It also prevents us from retrospectively tagging a resource as problematic or insufficient, should the research process reveal issues with a particular resource.

Until recently challenges in resource identification and methodological reporting have been largely anecdotal, but several efforts have begun to characterize this problem and enact solutions. The National Centre for the Replacement, Refinement and Reduction of Animals in Research (NC3R) evaluated methodological reporting in the literature for in vivo studies using rodent models or non-human primates. They examined 271 publications and reported that only 
65

66

67

68

69

70

71

72

73

74

75

76

77

78

79

80

81

82

83

84

85

86

87

88

89

90

91

92

93

94

95

96

97

98

99

100

101

102

103

104

105

106

107

108

109

\section{Methods}

111 Journal selection and classification organizations.

$60 \%$ of the articles included information about the number and characteristics of the animals (strain, sex, age, weight) and approximately $30 \%$ of the articles lacked detailed descriptions of the statistical analyses used (Kilkenny et al. 2009). Based on this study, the ARRIVE guidelines (http://www.nc3rs.org.uk/page.asp?id=1357) were developed for reporting of in vivo experiments pertaining to animal research. Other domain specific standards have been published such as the Minimum information about a protein affinity reagent (MIAPAR) (Bourbeillon et al. 2010) and the high-profile communication from Nature to address concerns regarding research reproducibility where they offered improved standards for reporting life science research (http://www.nature.com/authors/policies/reporting.pdf). The Neuroscience Information Framework (NIF; http://neuinfo.org) specifically developed the Antibody Registry as a means to aid identification of antibodies within published studies, based on a small pilot study which showed that $>50 \%$ of antibodies could not be identified conclusively within published papers. (Bandrowski et al., in preparation). ISA-TAB provides a generic, tabular format, which contains metadata standards to facilitate data collection, management, and reuse (Sansone et al. 2012; Sansone 2013; Thomas et al. 2013). To promote scientific reproducibility, the Force11 community has published a set of recommendations for minimal data standards for biomedical research (Link Animal Models to Human Disease, LAMHDI; 2012) and published a manifesto to improve research communication (Phil Bourne, Tim Clark, Robert Dale, Anita de Waard, Ivan Herman, Eduard Hovy 2011). The BioSharing initiative (www.biosharing.org) contains a large registry of community standards for structuring and curating datasets and has made significant strides towards the standardization of data via its multiple partnerships with journals and other

While the work highlighted above has offered guidance based on the perceived problem of inadequate methodological reporting, the fundamental issue of material resource identification has yet to be specifically characterized using a rigorous scientific approach. It is our belief that unless researchers can access the specific research materials used in published research, they will continue to struggle to accurately replicate and extend the findings of their peers. Until our long held assumptions about a lack of unique identifiability of resources are confirmed with quantitative data, this problem is unlikely to pique the interest of funding agencies, vendors, publishers, and journals, who are in a position to facilitate reform. To this end, we report here an experiment to quantify the extent to which material resources reported in the biomedical literature can be uniquely identified. We evaluated 238 journal articles from five biomedical research sub-disciplines, including Neuroscience, Developmental Biology, Immunology, Cell and Molecular Biology, and General Biology. Target journals were selected from each category to include a representative variety of publishers, impact factors, and stringencies with respect to materials and methods reporting guidelines. In each article, we tracked reporting of five types of resources: (1) model organisms (mouse, rat, zebrafish, worm, fly, frog, and yeast); (2) antibodies; (3) knockdown reagents (morpholinos or RNAi); (4) DNA constructs; and (5) cell lines. We developed a detailed set of evaluation criteria for each resource type and applied them to determine the identifiability of over 1,700 individual resources referenced in our corpus. The results of this experiment quantify a profound lack of unique identification of research resources in the biomedical literature across disciplines and resource types. Based on these results and the insights gained in performing this experiment, we provide recommendations for how research resource identification can be improved by implementing simple but effective solutions throughout the scientific communication cycle. 
The core of our evaluated corpus was comprised of articles from a set of target journals

113 that varied across three features: research discipline, impact factor, and reporting guideline

114 requirements. For research discipline selection, we followed the Institute for Scientific

115 Information (ISI) categorization and selected five journals from Cell Biology, Developmental

116 Biology, Immunology, and Neuroscience. In addition, a non-ISI category (General Biology) was

117 included to cover multidisciplinary journals such as Science, Nature, and PLoS Biology. Within

118 each discipline, care was taken to include journals with a range of impact factors as reported in

119 the Journal Citation Report from 2011 (Thomson Reuters 2011). Journals were binned into three

120 categories (high, mid, and low) based on whether their impact factor fell into the top, middle, or

121 lowest third for their discipline in this report. Finally, we selected journals that varied in the

122 stringency of their recommendations for reporting data about material resources. Journals were

123 assigned to one of three categories: (1) Stringent if the journal required detailed information or

124 specific identifiers to reference materials reported in the manuscript (e.g. required catalog

125 numbers for antibodies); (2) Satisfactory if the journal provided only limited recommendations

126 for structured reporting or resource identifiers, but did not restrict space allocated for this

127 information; and (3) Loose where minimal or no reporting requirements for materials and

128 methods were provided, and/or the length of material reporting space was restricted. Note that

129 these guidelines were the ones in effect at the time of manuscript selection (January 18, 2013).

\section{Article selection}

131 Articles in the core collection of our corpus were selected randomly by performing a PubMed search filtered for each journal and using the first five publications returned on January 18, 2013 (all publications were from 2012-2013). This approach was adequate for all journals except Nature and Science, which cover a very general scientific spectrum such that top PubMed hits often failed to include the resource types evaluated in our study. For these journals, the most recent articles that were likely to contain our resources were selected directly from the publisher's website. Recent publications were chosen for our corpus deliberately to reduce the chance that they had been curated by a model organism database (MOD) or other curatorial efforts, which could skew results by providing additional curated data not reported or accessible from the original article alone. NIF had also noted in a pilot project that the identifiability of reagents decreases over time, as commercial vendors eliminate products from their catalogs.

In addition to this core collection of 135 core articles, we added 86 additional publications to our study through a collaboration with the Zebrafish Information Network (ZFIN), who agreed to assess identifiability of reported resources according to our evaluation guidelines as part of their established curation pipeline. Finally, a set of 17 more articles from the Nathan Urban Laboratory at Carnegie Mellon University was included in our experiment. The Urban lab studies cellular and systems neuroscience, and extensively uses animal models and antibodies. These articles were included to explore how the thorough and structured documentation practices of this lab in its internal management of resource inventory and usage is reflected in its reporting of materials in the literature they produce. Articles from these additional ZFIN and Urban lab collections were also classified according to discipline and impact factor, so as to be included with our core collection in our factor analysis. In total, 238 manuscripts were analyzed from 84 journals. All of the articles contained at least one or more of the research resources we evaluated in this study. To ensure this was a sufficient number of papers, we did preliminary statistical analysis to determine that we could find statistical significance in the results. A list of the journals, domains, impact factors, and PubMed IDs, as well as complete dataset is available in Supplemental Table 1.

\section{Article Curation Workflow}


A team of three curators evaluated a selection of articles from the corpus, with each being reviewed by a single expert to identify and establish the identifiability of each documented resource. In addition, fly and zebrafish genetics experts curated the zebrafish and drosophila model organisms, respectively, as our primary curators did not have expertise in these areas. We performed spot-checking of the primary curation and issues found by the secondary evaluator were documented in the curation spreadsheets and updates were made to the curation guidelines. Where necessary, the curator used supplemental data and any referenced articles or publically accessible online data sources, dating as far back as necessary to find uniquely identifying information about a resource. This included vendor catalogs and a variety of experimental and resource databases, where identifying information was often resolvable based on information provided in a publication. More detailed evaluation criteria for unique identification of each resource type are described below. For a given article, evaluation of only the first five resources of each type was performed in the core publication collection. This was necessary as some papers referenced a cumbersome number of resources such as antibodies or RNAi oligos, which were typically reported to the same degree of rigor.

\section{Resource identification criteria}

175 Based on our extensive experience in working with these particular resources and on 176 consultation with several external experts, we developed a set of criteria to determine the ability

177 of each resource type to be 'uniquely identified'. Generally, 'unique identification' requires that a 178 specific resource can be obtained or created based on information provided in or resolvable from the publication directly, or resolvable through referenced literature, databases, or vendor sites. Below we outline some general and resource-type specific requirements for 'identifiability' applied in our evaluations.

\section{I. General Considerations}

183 Catalog Numbers

184 For commercial resources, provision of a catalog number and the name of the vendor that 185 resolves to a single offering uniquely identifies a resource. In the absence of a catalog number, if provision of only the vendor and resource name allows unambiguous resolution to a single offering, a resource is considered identifiable. For example, reporting "polyclonal anti-HDAC4 from Santa Cruz" resolves to a single antibody in the Santa Cruz catalog even without a catalog number. However, this is not ideal, because the catalog may expand to include additional polyclonal anti-HDAC4 antibodies in the future, which would render the resource unidentifiable. Additionally, catalog numbers are not stable as products are discontinued or sold; hence we also looked for a record of the antibody in the Antibody Registry (www.antibodyregistry.org), which provides stable IDs for antibody offers.

\section{Sequence Molecule Identification}

Sequence identification is a central aspect of identifiability for many resource types. Examples include specifying the sequence of an immunogenic peptide for a lab-sourced antibody, the sequence of a DNA insert of a construct, or the sequence of a transgene incorporated into the genome of an organism or cell line. In such cases, these sequences need to be resolvable to known information about the specific nucleic acid or peptide sequence to support identifiability of the resource to which they are related. Criteria that establish resolution of a sequence in support of identifying a dependent resource include: (1) directly providing the full sequence; (2) referencing a resource from which the sequence can be determined (to the extent that it is known) - e.g. by providing a gene ID or accession number that can be looked up and a sequence determined; (3) when precise/complete sequence information does not exist, a sequence should 
206

207

208

209

210

211

212

213

214

215

216

217

218

219

220

221

222

223

224

225

226

227

228

229

230

231

232

233

234

235

236

237

238

239

240

241

242

\section{Cell Lines}

244 For standard publically available lines, an unambiguous name or identifier is required as well as a

245

246

247

248

249

250

251

252

be tied to some other unique entity, such as a single, unique source and procedure through which the physical sequence can be obtained/replicated (e.g. primers and a specific source of template DNA such as a uniquely identified cell type or biological sample). The requirement for complete resolution to a specific sequence is not absolute as it is sometimes the case that this information is not known, and for some resource types a complete sequence may not be required to be considered uniquely identifiable. One recurring theme we encountered in our study was authors referencing a gene name or sequence to identify cDNA or a peptide related to the gene. This can be problematic, as specification of a gene sequence may not be sufficient to resolve a single cDNA or peptide sequence. This is because a single gene may resolve to many different transcripts or peptides (e.g. through alternative splicing), which can prevent unambiguous resolution of a gene sequence to a cDNA or peptide sequence.

\section{Specific Resource Identification Criteria Antibodies}

Unique antibody identification required at least one of the following: (1) an identifier resolving to a universal registry/database identifier such as the Antibody Registry (www.antibodyregistry.org) or eagle-i repository (http://www.eagle-i.net), or a vendor name and catalog number for resolving to a single offering; (2) for antibodies not publicly available, sufficient protocol details on production of the antibody so as to allow reproduction. This detail minimally includes specifying the host organism and identity of the immunogen used. For peptide immunogens, criteria for sequence identification above apply, i.e. that an immunogenic protein or peptide resolves to single gene product sequence. Note that the criteria for identifiability do not include the lot or batch number, although a case could be made for this level of granularity.

\section{Organisms}

For 'wild-type' organism strains, an unambiguous name or identifier, such as a stock number, the official International Mouse Strain Resource (IMSR) name or a MOD number, is required as well as a source vendor, repository, or lab. For genetically modified strains, identifiability requires reporting or reference to all genotype information known, including genetic background and breeding information, and precise alterations identified in or introduced into the genome (including known sequence, genomic location, and zygosity of alterations). For random transgene insertions, it is not required that genomic location of insertion(s) is known, but precise sequence of inserted sequence should be unambiguously resolvable according to sequence identification criteria above. For targeted alterations, genomic context of the targeted locus and the precise alterations to the locus should be specified according to sequence identification criteria above. This information can be provided directly, or through reference to a MOD record or catalog offering where such information is available. The MODs provide specific nomenclature guidelines that are consistent with these views. source for the line (e.g. a vendor or repository). This information should resolve to data about the organismal source and line establishment procedures. For example, a common cell line reported that can be obtained from ATCC would be considered identifiable, however if only the name of the line is mentioned without any other identifying information then it is considered unidentifiable. For novel lab-generated cell lines, an organismal source (species and known genotype information, anatomical entity of origin, developmental stage of origin) and any relevant procedures applied to establish a stable lineage of cells. Additionally, some indication of passage number is recommended but not strictly required. For genetically modified lines, 
253 identifiability criteria are analogous to those for genetically modified organisms, including

254 genomic location and zygosity or copy number of modifications where this information is known.

255 Constructs

256 Construct backbone should be unambiguously identified and resolvable to a complete vector

257 sequence (typically through a vendor or repository). The sequence of construct inserts should be

258 identifiable according to sequence identification criteria above. Most expression constructs

259 incorporate cDNA - so it is particularly important that the exons included in this insert are

260 resolvable when more than one splice variant exists for a gene transcript. This means that

261 specifying the name of a gene or a protein expressed may not be sufficient if this does not allow

262 for unambiguous resolution to a cDNA sequence. Identification does not require precise

263 description of MCS restriction sites used for cloning, but this information is encouraged. Relative

264 location and sequence of epitope tags and regulatory sequences (promoters, enhancers, etc)

265 should be specified (e.g. 'N-terminal dual FLAG tag' is sufficient). For example, referencing the

266 accession number and the vector backbone is sufficient to identify the construct, as in: "for the

267 full-length Dichaete construct, the insert was amplified from the full-length cDNA clone

268 (GenBank accession X96419 and cloned into the HindIII and KpnI sites of pBluescript II KS(!)”

269 (Shen, Aleksic, and Russell 2013). However, in most constructs, such level of detail is omitted.

\section{Knockdown Reagents}

271 Identifiability requires specific and complete sequence identification according to the criteria

272 outlined above. This will typically be direct reporting of the sequence, as these are generally short

273 oligos. For example, this text provided in the method section was considered identifiable: "The

274 DNA target sequence for the rat Egr-2 (NM_053633.1) gene was

275 CAGGAUCCUUCAGCAUUCUTT" (Yan et al. 2013). In cases where sequence information was

276 not provided, the reagent was considered unidentifiable.

277 Statistical analysis

278 Since the data was binomial in that each resource was either identifiable or not, we used a

279 binomial confidence interval strategy for calculating upper and lower 95\% confidence intervals

280 (CI) (http://www.biyee.net/data-solution/resources/binomial-confidence-interval-calculator.aspx).

281 Error bars for the corresponding 95\% CI are displayed on the graphs. Statistical significance was

282 determined by calculating the $\mathrm{z}$-score.

\section{Results and Discussion}

284 The goal of our study was to determine the proportion of research resources of five common

285 types that can be uniquely identified as reported in the literature. 'Unique identification' requires

286 that a resource can be obtained or re-created based on information provided in or resolvable from

287 a publication. The criteria for identifiability were established a reasonable level of granularity,

288 recognizing that finer levels, e.g., lot or litter number, may be possible. Establishing

289 identifiability criteria was central to our effort, and these criteria are complex and varied between

290 resource types as described in the Methods section. The results of our study provide

291 quantification of this problem in the literature. In total, only 54\% (922/1703) of evaluated

292 resources were uniquely identifiable. Considerable variability was found across resource types

293 (Figure 1A), which may result from the inherent differences in the attributes relevant to their

294 identification, or from the level of external support for applying identifiers and metadata for their

295 unique identification. In addition, the level of identifiability for each resource type is tied directly 
296

297

298

299

300

301

302

303

304

305

306

307

308

309

310

311

312

313

314

315

316

317

318

319

320

321

322

323

324

325

326

327

328

329

330

331

332

333

334

335

336

337

338

339

340

341

342

343 to the stringency of the criteria that were separately developed for each, which are unavoidably exposed to some degree of subjectivity.

\section{Antibodies}

Antibody reagents represent one of the most challenging and important resource types to adequately identify, given their ubiquitous use, expense to create, and condition-specific efficacy. The most common issue with reporting of antibodies was a lack of catalog number (for commercial antibodies) or a lack of reference to the immunogen used to generate the antibody (for non-commercial antibodies). A separate analysis of commercial versus non-commercial (e.g lab-made) antibodies showed an average of $46 \%$ of commercial antibodies, and similarly, $43 \%$ of non-commercial antibodies were identifiable. While commercial suppliers do an acceptable job of providing basic metadata about their offerings (for example, see http://datasheets.scbt.com/sc-546.pdf), the market is flooded with products of variable quality metadata. In practice, the literature is where most scientists look when searching for the right antibody for their work, as evidenced by a marketing report from 1 Degree Bio (http://1degreebio.org/) showing 63\% of researchers use journal references to guide antibody selection (A. Hodgson, unpublished data). This makes it all the more troubling that only $44 \%$ of antibodies evaluated in our study could be uniquely identified (Figure 1B). While reporting of a catalog number alone is considered sufficient for unique identification of a commercially available antibody, we found they were provided for only $27 \%$ of antibodies we evaluated.

A likely reason for the shortcoming in commercial antibody identification may be that journal reporting guidelines rarely require catalog numbers be reported for antibodies (or any other reagent type for that matter). More commonly, only a name and location of a manufacturer are required. For example, the journal "Immunology" simply states: "Materials and Methods: sufficient information must be included to permit repetition of experimental work. For specialist equipment and materials the manufacturer (and if possible their location) should be stated." (Wiley Online Publishing). By contrast, the Journal of Comparative Neuroscience (JCN) is one of the rare journals that do require more precise reporting of antibody metadata, including their catalog number. An extensive evaluation of 6,510 antibodies in the JCN Antibody Database (Wiley Online Publishing 2013) revealed that a catalog number was reported in over $90 \%$ of the antibodies captured in their database (Bandrowski et al., in preparation, and re-evaluated in this study). This highlights how simple solution such as requiring catalog number reporting can vastly improve resource identification in the literature.

Notably, as more data is becoming available about protein structure, localization, and function, the identity of peptide immunogens and epitopes used in creating an antibody becomes increasingly valuable for explaining its performance in different applications. Identification and tracking of immunogens is one area where there is considerable room for improvement among vendors and resource databases. Efforts such as the Immune Epitope Database (IEDB) (http://www.iedb.org/), a manually curated repository of immunological data about epitope recognition, can be looked to for guidance in how to capture and represent relevant data about such epitopes. The IEDB curates papers that report discovery of new epitopes and even in this very specific use case where the goal is report on the specific epitope, only $81 \%$ of the epitopes they curated had the epitope sequence provided in the published manuscript (R Vita, unpublished data).

\section{Cell lines}

A source for cell lines was rarely reported and the lack of source was most common factor for their low identifiability in our study. For commonly used, unmodified lines such as HEK293T cells, our guidelines required a source be provided in addition to the line name. This information 
344 was deemed important given the tendency of lines propagated in isolation to diverge genetically

345 through continuous passages (Hughes et al. 2007). There are increasingly documented

346 occurrences of cell line misidentification and contamination, as highlighted by the infamous

347 HeLa contamination statistics (Gartler 1968) and other cell line contaminations (Phuchareon et al.

348 2009). Simply reporting the name of the line without a source fails to provide any information on

349 the history and integrity of the line. For lab-generated or genetically modified cell lines not

350 available from a public source, identification required a basic description of the line's

351 establishment procedure, its anatomical source, and/or the precise genetic modifications made

352 (see details in Methods section).

353

354

355

356

357

358

359

360

361

362

363

364

365

366

367

368

369

370

371

372

373

374

375

Based on these criteria, the identifiability of cell lines was comparable to that for antibodies, averaging 43\% across all disciplines (Figure 1C). A notable difference was found for cell line identifiability between our lowest and highest reporting disciplines - General Biology (0\% identifiable) and Immunology (88\% identifiable). This may reflect the tendency for less rigorous reporting requirements and reduced space allocation for methods that are common in high-profile journals we included in this category (e.g. Nature, Science). By contrast, the majority of cell lines reported in Immunology papers adequately referenced either the lab, investigator, or commercial supplier that provided the cell line, which may indicate more rigorous conventions for sharing and attribution for cell lines in this community; however, due to the low number of cell lines evaluated in immunology journals in this study, we cannot make this conclusion.

An important aspect of cell lines that we found highly neglected in literature reporting was passage number. This attribute provides an important metric to gauge the integrity of a cell line sample, and how likely it is to be faithfully reflected in another sample. We found such information to be rarely reported in our study, and thus did not require it in addition to a source for identifiability. But we highly recommend more attention be paid to tracking and reporting this important attribute in the literature. This practice is particularly important for lines propagated in research labs, as a survey on cell line usage reported that $35 \%$ of researchers use cell lines obtained from another lab rather than a cell line repository (Buehring, Eby, and Eby). Tracking passage number and contamination is a lower priority in these labs compared to commercial repositories, such that the use of genetically or compositionally divergent samples of the same line is likely to be a significant contributor to difficulties in reproducing cell-line based research. Towards this end, a guideline has been published to check for contamination and authenticity of cell lines (Capes-Davis et al. 2010).

\section{DNA Constructs}

377 Unique identification of constructs was the lowest amongst all resource types examined, 378 on average 25\% were identifiable, due to lack of reporting of sequence or other identifying information (Figure 1D). This was likely due to the dependency of identification on reporting a complete or approximated sequence, and the lack of incentive, guidelines, or technical support for providing such metadata. While many construct backbones are obtained from commercial manufacturers where the relevant sequence information is provided, the valuable component of a construct are the gene(s) that have been sub-cloned in by a researcher. Access to this sequence information is critical in order to reproduce the experiment or fully utilize these resources, but it is rarely directly reported in full. While resources like Addgene and PlasmID provide detailed information about constructs and the relevant gene components, submission of plasmids to such repositories is infrequent, as we found less than $10 \%$ of non-commercial plasmids reported in our corpus to be present in such repositories. In cases where primer sets were used to generate a construct insert, we often found that the primer sequences were reported; yet the specific and 391 complete sequence of the amplified template was rarely specified. In such cases, it is not possible to determine the sequence of the product cloned into a construct. 
392

393

394

395

396

397

398

399

400

401

402

403

404

405

406

407

408

409

410

411

412

413

414

415

416

417

418

419

420

421

422

423

424

425

426

427

428

429

430

431

432

433

434

435

436

437

\section{Gene Knockdown Reagents}

Knockdown reagents were significantly more identifiable compared to the former resource types mentioned above, at $83 \%$ (Figure $1 \mathrm{E}$ ). Knockdown reagents are frequently used, in particular in Cell and Developmental Biology (Harborth et al. 2001; Nasevicius and Ekker 2000). Identifiability of knockdown reagents was the highest amongst resource types. This is likely due to the fact that knockdown reagents tend to be comprised of short, and therefore easy to include, sequence information. Additionally, editors often require reporting of sequences for custom reagents, as this information is critical to understanding and verifying the reagent function. MODs also keep track of these sequences as they curate papers. The majority of knockdown reagents that were curated in this study were from Developmental Biology journals, which also had the lowest number of identifiable reagents compared to other fields. Knowing the exact sequence used is necessary to reproduce the experiment, and concentration and experimental details are similarly important to determine off-target effects.

\section{Organisms}

Organisms showed a relatively high identifiability of 77\% (Figure 1F). Amongst organisms, yeast were the most identifiable (100\%, albeit there were only 5 strains analyzed from one paper), followed by zebrafish (87\%), flies (80\%), mice (67\%), and rats (60\%). Worms and frogs were the least identifiable, at $58 \%, 33 \%$, and $0 \%$, respectively. The identification of transgenic organisms was higher, with $83 \%$ of transgenic organisms being identifiable compared to $46 \%$ of non-transgenic wild type strains. The higher identifiability may be due to the fact that $56 \%$ of the transgenic strains we analyzed had already been curated by a MOD, because the organisms reported in our corpus were previously reported in an earlier publication that had been curated by a MOD. Indeed, identifiability of organisms not found in a MOD was considerably lower at $60 \%$. The MODs review the current literature and annotate information about genetic modifications used in transgenic strains, phenotypes, gene expression, etc., in addition to other relevant types of information pertaining to the organisms (Bradford et al. 2011; Bowes et al. 2010; Yook et al. 2012; Marygold et al. 2013; Laulederkind et al. 2013; Bult et al. 2013). While it is reassuring that these specific strains have been previously curated via earlier publications, it often requires the curator to dig through many publications or to contact the authors directly. ZFIN determined that over a two-month period, they had to contact $29 \%$ of authors to properly curate the resources reported in their manuscript.

Comparing organism identification between disciplines, we noted that they were considerably less identifiable in Neuroscience papers (46\%) relative to other domains. A likely explanation is that non-transgenic animals are commonly used in neuroscience assays such as electrophysiology studies (26 out of 62 organisms analyzed were non-transgenic). Identification of such commercially available strains faces similar problems as standard cell lines, where a source is required to allow some historical information to be obtained about propagation/breeding. Indeed, it has been reported that there are many variations between wild type strains of model organisms (Portelli et al. 2009; Sandberg et al. 2000; Wahlsten 1987), and variations between suppliers (Ezerman and Kromer 1985).

\section{Domain considerations}

We further examined if the unique identification of resources differed between sub-disciplines of biomedical research (Table 1 ). While no discipline was consistently above or below average with respect to identification of the resources, Developmental Biology, General Biology, and Immunology were generally above average compared to the other fields. The identification of cell lines was highest in Immunology papers, which was significantly different

PeerJ reviewing PDF | (v2013:05:544:1:0:NEW 1 Aug 2013) 
438 from Cell and General Biology papers, and papers from the "other" category, even though there

439 was a small sample size (16 out of 104 total cell lines were from Immunology journals). By

440 contrast, no cell lines were identifiable in the General Biology papers, which was significantly

441 lower compared to all disciplines except the "other” category. However, General Biology journals

442 boasted the highest percentage of identifiable constructs in papers at 59\%, which was a

443 significantly better compared to the other disciplines except Immunology. It is notable that

444 identification of resources for Neuroscience was below average compared to the other fields for

445 all resources except cell lines. Of note, identification of organisms in Neuroscience journals was

446 significantly less than all other disciplines (30 out of 62 organisms were identifiable). Overall,

447 there was not a consistent trend between scientific sub-domains with respect to identifiability of

448 resources (Figure 1B-F).

449 Impact factor considerations

450 We next examined whether identification of resources differed among journals across a

451 range of impact factors. We found that resource identification did not vary with journal impact

452 factor, as revealed by the lack of correlation in scatter plot analysis (Figure 2A-E).

453 Analysis by reporting requirements

$454 \quad$ Very few journals were considered to have stringent reporting requirements, and amongst

455 those, it was surprising to note that the identification of the resources did not appear improved

456 above journals with satisfactory or loose reporting requirements. Identification of cell lines was

457 especially poor in journals with satisfactory reporting guidelines ( 0 out of 21 were identifiable,

458 from 10 articles analyzed), and overall, the identification of the resources was the poorest in

459 journals with highest reporting requirements (an average of $45 \%$ were identifiable in journals

460 with stringent reporting requirements, while resources from journals with satisfactory and loose

461 were on average $61 \%$ and 55\% identifiable, respectively; Figure 3). On average, journals with

462 loose reporting requirements had a significantly higher percentage of identifiable resources

463 compared to journals with stringent reporting requirements.

464 With most journals having a low or mid-level impact factor (i.e. a skewed distribution), 465 the majority of high identifiability therefore comes from these lower profile journals. This is an

466 encouraging result, because it means that the lion's share of the publishing world has already

467 demonstrated a capability of producing identifiable resources. It is especially important to not

468 overlook these higher volume lower-cited journals to produce quality metadata about research

469 resources. Additionally, higher impact journals tend to de-emphasize methods over other sections.

470 Therefore, what is needed is to incentivize all journals to do better with respect to identifiability.

471 Lab documentation vs. publications

472 For the Urban lab publications that we evaluated, only $44 \%$ of the antibodies used were

473 identifiable (out of 9 total antibodies from 5 papers), and $47 \%$ of the organisms were identifiable

474 (out of 17 organisms from 17 papers). We note that this lab internally keeps highly structured

475 notes and metadata about their resources in the lab; after analyzing their internal notes, $100 \%$ of

476 antibodies and $100 \%$ of organisms were identifiable using our criteria. However, despite this

477 information being tracked extensively within the lab, these details did not make it into their

478 publications. It does suggest, however, that the information is potentially recoverable, if practices

479 to make resources identifiable are implemented.

480 Evaluation Criteria and Workflow

481 A core challenge of designing this experiment was determining evaluation criteria that

482 were precise enough to allow for reproducible determination of reported resource identifiability. 
483 For simplicity, we used a binary classification for the data analysis, but in reality the amount of

484 information pertaining to resource identification was incremental. Crafting of these criteria

485 required careful consideration of each resource type, including how they are generated and

486 acquired and the particular aspects of each that are important in the context of experimental

487 reproducibility. This was particularly complex for resources whose identification required

488 sequence information relating to a target or part of the resource, as different applications may

489 require different degrees of specificity. Despite the abundance of public databases that provide

490 identifiers for biological sequences, we found a reluctance of authors to reference such IDs when

491 documenting reagents such as constructs or antibodies. This may point to a lack of awareness, a

492 lack of incentive, or a lack of means for the journals and authors to use existing resources to

493 supply uniquely identifiable information. Each problem is likely to have its own set of solutions,

494 which we discuss in our recommendations below.

495 To ensure their consistent application, criteria and evaluation workflows were centrally

496 documented, performed, and evaluated performed by expert biocurators. These results support

497 the specificity and reproducibility of our guidelines, which we hope will serve to inform reporting

498 requirements of publishers and the development of support platforms for authors.

\section{Conclusions}

500

501

502

503

504

505

506

507

508

509

510

511

512

513

514

515

516

517

518

519

520

521

522

523

524

525

526

527

528

529

Improving reporting guidelines for authors is an important step towards addressing this problem. Very few journals (only 5/83) had high stringency guidelines by our definition. Higher impact journals like Science and Nature tended to have looser reporting requirements, usually due to space limitations in the journal and often required reference to previously published methods. It has also been previously noted that higher impact journals have a higher retraction rate (Fang \& Casadevall 2011). The Journal of Comparative Neurology has stringent reporting standards for materials and methods, requiring that sources for all materials and equipment, sequence information for nucleic acids and peptides, and immunogen and catalog number for antibodies be reported. It is our hope that other journals will follow suit. That said, we found that antibody identifiability in the Journal of Comparative Neurology was only slightly higher than average across all journals (58\% in JCN vs. 44\% overall). Our findings are also much lower than the percentage calculated from the JCN database above, perhaps due to lack of compliance by authors or lack of enforcement by reviewers. Based on the sampling that we have, there does not seem to be any relationship between reporting guidelines and identifiability. One might ask, how can this be? The reality is that having quality guidelines for authors is only one part of the solution. For example, Mike Taylor writes about how the peer review process fails to enable trustworthy science (Taylor 2013).

The solution to improving resource identifiability and therefore scientific reproduciblity needs to be a partnership between all participants in the scientific process, and deficiencies in awareness and difficulties coordinating across these stakeholders is at the root of the problem. Better tracking of research resources by researchers during the course of research can facilitate sharing of information with databases and at publication time. Electronic lab notebooks and management software (Machina and Wild 2013; Hrynaszkiewicz 2012), or resource sharing repositories such as the eagle-i Network (www.eagle-i.net) (Vasilevsky et al. 2012) or the Neuroscience Information Framework (http://www.neuinfo.org/) (Bandrowski et al. 2012) enable creation of stable identifiers and structured tracking of information. The MODs have recommended nomenclature standards for organisms, but these are not always adhered to (RGD 2005; MGI 2013; ZFIN 2013; Flybase 2013). In an ideal situation, authors would report the unique ID pertaining to the model organism directly in the publication by having their ID assigned and nomenclature approved prior to publication. Then a direct link and easy access to

PeerJ reviewing PDF | (v2013:05:544:1:0:NEW 1 Aug 2013) 
530 the information to researchers who are attempting to understand or reproduce an experiment can

531 be made available. In addition, this can facilitate text-mining and machine processing using

532 automated agents that recognize these IDs. Journal editors should better detail reporting

533 requirements, such as in the recent communiqué from Nature

534 (http://www.nature.com/authors/policies/reporting.pdf). Publishers also need functionality to

535 identify resources at the time of submission. Tools such as the DOMEO Toolkit allow for

536 semantic markup of papers (Ciccarese, Ocana, and Clark 2012) and can be utilized during the

537 submission process whereby researchers can easily check the identifiability of the resources

538 found in their paper. Vendors, if more aware of how their products are being referenced in the

539 literature and databases, may tend towards better and more stable catalog schemes as well as to

540 integrate the added knowledge being captured in external resources. Finally, researchers can be

541 attributed for their resources so that they would be incentivized to uniquely identify and share

542 them. Recent changes to the NSF biosketch highlight a specific area where uniquely identifying

543 such resources can have a positive influence on the evaluation of one's scholarly activities.

544 Similarly, the Bioresource Research Impact Factor (BRIF) (Mabile et al. 2013) provides

545 attribution for use and sharing of resources. Unique reference of resources through databases

546 such as the Antibody Registry, eagle-i or MODs can facilitate this process. Finally, researchers

547 need to know where the information in their favorite online resources comes from - the literature

548 and the biocurators that curate their papers and datasets. Identifiability is just as important in the

549 context of data sets, and given the significant effort being made to make informatics analyses

550 reproducible (http://www.runmycode.org/CompanionSite/) and data sets available (dryad.org), it

551 is ironic that in some cases the original data itself may not be reproducible simply because the

552 antibody used to generate the data was never specified.

553 Scientific reproducibility is dependent on many attributes of the scientific method. Being

554 able to the uniquely identify the resources used in the experiments is only one of these attributes

555 - it just happens to be the easiest one to accomplish. We hope that this study insights authors,

556 reviewers, editors, vendors, and publishers to work together to realize this common goal.

\section{Acknowledgements}

558 We would like to acknowledge Robin Champieux for her help with the experimental design, John

559 Campbell for his help with data and statistical analysis and discussion, Scott Hoffmann for his

560 help with the data analysis and figure preparation, Alex Hodgson for sharing the antibody market

561 analysis and manuscript review, Nathan Urban for discussions and sharing information on lab

562 internal databases and notes, and Randi Vita for manuscript review and for sharing the IEDB

563 data, Ceri Van Slkye for her help with analyzing the yeast strains, and Anita de Waard, Maryann

564 Martone, and Anita Bandrowski for discussion and manuscript review.

566 Funding support

567 OHSU acknowledges the support of the OHSU Library and \#1R24OD011883-01 from the NIH

568 office of the Director. The Zebrafish Information Network and Flybase are funded by the

569 National Human Genome Research Institute (P41 HG002659 and P41 HG000739, respectively.

570 Shreejoy Tripathy of the Urban Lab is funded by an NSF graduate research fellowship and a RK

571 Mellon Foundation fellowship. Greg LaRoca is funded by NIH grants R01DC005798 and

572 R01DC011184.

\section{References:}

574 Bandrowski, A E, J Cachat, Y Li, H M Müller, P W Sternberg, P Ciccarese, T Clark, et al. 2012.

575 "A Hybrid Human and Machine Resource Curation Pipeline for the Neuroscience

576 Information Framework." Database : the Journal of Biological Databases and Curation 
2012 (January): bas005. doi:10.1093/database/bas005.

578

579 http://www.pubmedcentral.nih.gov/articlerender.fcgi?

580 artid $=3308161 \&$ tool=pmcentrez\&rendertype=abstract.

581

582

583

584

585

586

587

588

589

590

591

592

593

594

595

596

597

598

599

600

601

602

603

604

605

606

607

608

609

610

611

612

613

614

615

616

617

618

619

620

621

622

Begley, C Glenn, and Lee M Ellis. 2012. "Drug Development: Raise Standards for Preclinical Cancer Research.” Nature 483 (7391) (March 29): 531-3. doi:10.1038/483531a. http://www.nature.com.liboff.ohsu.edu/nature/journal/v483/n7391/full/483531a.html.

Bourbeillon, Julie, Sandra Orchard, Itai Benhar, Carl Borrebaeck, Antoine de Daruvar, Stefan Dübel, Ronald Frank, et al. 2010. "Minimum Information About a Protein Affinity Reagent (MIAPAR).” Nature Biotechnology 28 (7) (July): 650-3. doi:10.1038/nbt0710-650. http://dx.doi.org/10.1038/nbt0710-650.

Bowes, Jeff B, Kevin A Snyder, Erik Segerdell, Chris J Jarabek, Kenan Azam, Aaron M Zorn, and Peter D Vize. 2010. "Xenbase: Gene Expression and Improved Integration.” Nucleic Acids Research 38 (Database issue) (January): D607-12. doi:10.1093/nar/gkp953. http://www.pubmedcentral.nih.gov/articlerender.fcgi? artid=2808955\&tool=pmcentrez\&rendertype=abstract.

Bradford, Yvonne, Tom Conlin, Nathan Dunn, David Fashena, Ken Frazer, Douglas G Howe, Jonathan Knight, et al. 2011. "ZFIN: Enhancements and Updates to the Zebrafish Model Organism Database.” Nucleic Acids Research 39 (Database issue) (January): D822-9. doi:10.1093/nar/gkq1077. http://www.pubmedcentral.nih.gov/articlerender.fcgi? artid=3013679\&tool=pmcentrez\&rendertype=abstract.

Buehring, Gertrude Case, Elizabeth A Eby, and Michael J Eby. "Cell Line Cross-contamination: How Aware Are Mammalian Cell Culturists of the Problem and How to Monitor It?” In Vitro Cellular \& Developmental Biology. Animal 40 (7): 211-5. doi:10.1290/1543-706X(2004)40<211:CLCHAA>2.0.CO;2. http://www.ncbi.nlm.nih.gov/pubmed/15638703.

Bult, Carol J, Janan T Eppig, Judith A Blake, James A Kadin, and Joel E Richardson. 2013. "The Mouse Genome Database: Genotypes, Phenotypes, and Models of Human Disease.” Nucleic Acids Research 41 (Database issue) (January): D885-91. doi:10.1093/nar/gks1115. http://www.pubmedcentral.nih.gov/articlerender.fcgi? artid $=3531104 \&$ tool $=$ pmcentrez\&rendertype $=$ abstract.

Capes-Davis, Amanda, George Theodosopoulos, Isobel Atkin, Hans G Drexler, Arihiro Kohara, Roderick A F MacLeod, John R Masters, et al. 2010. "Check Your Cultures! A List of Cross-contaminated or Misidentified Cell Lines.” International Journal of Cancer. Journal International Du Cancer 127 (1) (July 1): 1-8. doi:10.1002/ijc.25242. http://www.ncbi.nlm.nih.gov/pubmed/20143388.

Ciccarese, Paolo, Marco Ocana, and Tim Clark. 2012. "Open Semantic Annotation of Scientific Publications Using DOMEO.” Journal of Biomedical Semantics 3 Suppl 1 (January): S1. doi:10.1186/2041-1480-3-S1-S1. http://www.pubmedcentral.nih.gov/articlerender.fcgi? artid=3337259\&tool=pmcentrez\&rendertype=abstract.

Cokol, Murat, Fatih Ozbay, and Raul Rodriguez-Esteban. 2008. "Retraction Rates Are on the Rise.” EMBO Reports 9 (1) (January): 2. doi:10.1038/sj.embor.7401143. http://dx.doi.org/10.1038/sj.embor.7401143.

Ezerman, E.B., and L.F. Kromer. 1985. "Outbred Sprague-Dawley Rats from Two Breeders Exhibit Different Incidences of Neuroanatomical Abnormalities Affecting the Primary Cerebellar Fissure.” Experimental Brain Research 59 (3) (August). doi:10.1007/BF00261354. http://link.springer.com/10.1007/BF00261354. 
623 Fang, Ferric C, and Arturo Casadevall. 2011. "Retracted Science and the Retraction Index."

624 Infection and Immunity 79 (10) (October 1): 3855-9. doi:10.1128/IAI.05661-11.

625 http://iai.asm.org/content/79/10/3855.full.

626 Flybase. 2013. "FlyBase: Genetic Nomenclature for Drosophila Melanogaster.”

627 http://flybase.org/static_pages/docs/nomenclature/nomenclature3.html.

628 Gartler, S M. 1968. “Apparent Hela Cell Contamination of Human Heteroploid Cell Lines.”

$629 \quad$ Nature 217 (5130) (February 24): 750-1. http://www.ncbi.nlm.nih.gov/pubmed/5641128.

630 Haendel, Melissa A, Nicole A Vasilevsky, and Jacqueline A Wirz. 2012. "Dealing with Data: a

631 Case Study on Information and Data Management Literacy.” PLoS Biology 10 (5) (January):

632 e1001339. doi:10.1371/journal.pbio.1001339.

633 http://dx.plos.org/10.1371/journal.pbio.1001339.

634 Harborth, J, S M Elbashir, K Bechert, T Tuschl, and K Weber. 2001. "Identification of Essential

635

636

637

638

639

640

641

642

643

644

645

646

647

648

649

650

651

652

653

654

655

656

657

658

659

660

661

662

663

664

665

666

667

668

669 Genes in Cultured Mammalian Cells Using Small Interfering RNAs.” Journal of Cell Science 114 (Pt 24) (December): 4557-65. http://www.ncbi.nlm.nih.gov/pubmed/11792820.

Hrynaszkiewicz, Iain. 2012. "LabArchives and BioMed Central: a New Platform for Publishing Scientific Data - BioMed Central Blog."

http://blogs.biomedcentral.com/bmcblog/2012/04/04/labarchives-and-biomed-central-a-new -platform-for-publishing-scientific-data/.

Hughes, Peyton, Damian Marshall, Yvonne Reid, Helen Parkes, and Cohava Gelber. 2007. "The Costs of Using Unauthenticated, Over-passaged Cell Lines: How Much More Data Do We Need?” BioTechniques 43 (5) (November): 575, 577-8, 581-2 passim. http://www.ncbi.nlm.nih.gov/pubmed/18072586.

ISO 5725-1:1994. 1994. Accuracy (trueness and Precision) of Measurement Methods and Results. Geneva, Switzerland.

Kilkenny, Carol, Nick Parsons, Ed Kadyszewski, Michael F W Festing, Innes C Cuthill, Derek Fry, Jane Hutton, and Douglas G Altman. 2009. "Survey of the Quality of Experimental Design, Statistical Analysis and Reporting of Research Using Animals.” PloS One 4 (11) (January): e7824. doi:10.1371/journal.pone.0007824. http://www.pubmedcentral.nih.gov/articlerender.fcgi? artid $=2779358 \&$ tool $=$ pmcentrez\&rendertype $=$ abstract.

Laulederkind, Stanley J F, Weisong Liu, Jennifer R Smith, G Thomas Hayman, Shur-Jen Wang, Rajni Nigam, Victoria Petri, et al. 2013. "PhenoMiner: Quantitative Phenotype Curation at the Rat Genome Database.” Database : the Journal of Biological Databases and Curation 2013 (January): bat015. doi:10.1093/database/bat015. http://www.pubmedcentral.nih.gov/articlerender.fcgi? artid=3630803\&tool=pmcentrez\&rendertype=abstract.

Mabile, Laurence, Raymond Dalgleish, Gudmundur A Thorisson, Mylène Deschênes, Robert Hewitt, Jane Carpenter, Elena Bravo, et al. 2013. "Quantifying the Use of Bioresources for Promoting Their Sharing in Scientific Research.” GigaScience 2 (1) (May 1): 7. doi:10.1186/2047-217X-2-7. http://www.gigasciencejournal.com/content/2/1/7/abstract.

Machina, Hari K, and David J Wild. 2013. "Electronic Laboratory Notebooks Progress and Challenges in Implementation.” Journal of Laboratory Automation (April 16). doi:10.1177/2211068213484471. http://jla.sagepub.com/content/early/2013/04/12/2211068213484471.full.

Martone, Maryann and Link Animal Models to Human Disease (LAMHDI) Consoritum. 2012. "Publishing in the 21st Century: Minimal (really) Data Standards | FORCE11." http://www.force11.org/node/4145. 
Marygold, Steven J, Paul C Leyland, Ruth L Seal, Joshua L Goodman, Jim Thurmond, Victor B

671 Strelets, and Robert J Wilson. 2013. "FlyBase: Improvements to the Bibliography." Nucleic Acids Research 41 (Database issue) (January): D751-7. doi:10.1093/nar/gks1024. http://www.pubmedcentral.nih.gov/articlerender.fcgi? artid $=3531214 \&$ tool=pmcentrez\&rendertype $=$ abstract.

MGI. 2013. "MGI-Mouse Nomenclature Home Page.” http://www.informatics.jax.org/mgihome/nomen/index.shtml.

Nasevicius, A, and S C Ekker. 2000. "Effective Targeted Gene 'Knockdown' in Zebrafish.” Nature Genetics 26 (2) (October): 216-20. doi:10.1038/79951. http://www.ncbi.nlm.nih.gov/pubmed/11017081.

Phil Bourne, Tim Clark, Robert Dale, Anita de Waard, Ivan Herman, Eduard Hovy, David Shotton. 2011. "Force 11 Manifesto | FORCE11." http://www.force11.org/white_paper\#x1-200003.3.

Phuchareon, Janyaporn, Yoshihito Ohta, Jonathan M Woo, David W Eisele, and Osamu Tetsu. 2009. "Genetic Profiling Reveals Cross-contamination and Misidentification of 6 Adenoid Cystic Carcinoma Cell Lines: ACC2, ACC3, ACCM, ACCNS, ACCS and CAC2.” Ed. Ganesh Chandra Jagetia. PloS One 4 (6) (January): e6040. doi:10.1371/journal.pone.0006040. http://dx.plos.org/10.1371/journal.pone.0006040.

Portelli, Jeanelle, Najat Aourz, Dimitri De Bundel, Alfred Meurs, Ilse Smolders, Yvette Michotte, and Ralph Clinckers. 2009. "Intrastrain Differences in Seizure Susceptibility, Pharmacological Response and Basal Neurochemistry of Wistar Rats." Epilepsy Research 87 (2-3) (December): 234-46. doi:10.1016/j.eplepsyres.2009.09.009. http://dx.doi.org/10.1016/j.eplepsyres.2009.09.009.

RGD. 2005. "MGI_3.22 - Rules for Nomenclature of Genes, Genetic Markers, Alleles, and Mutations in Mouse and Rat Revised: Jan, 2005.” http://rgd.mcw.edu/nomen_rules.html.

Sandberg, R., R. Yasuda, D. G. Pankratz, T. A. Carter, J. A. Del Rio, L. Wodicka, M. Mayford, D. J. Lockhart, and C. Barlow. 2000. "From the Cover: Regional and Strain-specific Gene Expression Mapping in the Adult Mouse Brain.” Proceedings of the National Academy of Sciences 97 (20) (September 26): 11038-11043. doi:10.1073/pnas.97.20.11038. http://www.pnas.org/content/97/20/11038.short.

Sansone, Susanna-Assunta. 2013. "Our Roadmap to Engagement, Your Call : Scientific Data." http://blogs.nature.com/scientificdata/2013/05/13/our-roadmap-to-engagement-your-call/.

Sansone, Susanna-Assunta, Philippe Rocca-Serra, Dawn Field, Eamonn Maguire, Chris Taylor, Oliver Hofmann, Hong Fang, et al. 2012. "Toward Interoperable Bioscience Data.” Nature Genetics 44 (2) (February): 121-6. doi:10.1038/ng.1054. http://www.pubmedcentral.nih.gov/articlerender.fcgi? artid=3428019\&tool=pmcentrez\&rendertype=abstract.

Shen, Shih Pei, Jelena Aleksic, and Steven Russell. 2013. "Identifying Targets of the Sox Domain Protein Dichaete in the Drosophila CNS via Targeted Expression of Dominant Negative Proteins.” BMC Developmental Biology 13 (January): 1. doi:10.1186/1471-213X-13-1. http://www.pubmedcentral.nih.gov/articlerender.fcgi? artid=3541953\&tool=pmcentrez\&rendertype=abstract.

Taylor, Mike. 2013. "Peer Review Does Not Mean We Can Trust a Published Paper | Sauropod Vertebra Picture of the Week \#AcademicSpring on WordPress.com." http://svpow.com/2013/05/03/peer-review-does-not-mean-we-can-trust-a-published-paper/. Thomas, Dennis G, Sharon Gaheen, Stacey L Harper, Martin Fritts, Fred Klaessig, Elizabeth Hahn-Dantona, David Paik, et al. 2013. "ISA-TAB-Nano: a Specification for Sharing 
Thomson Reuters. 2011. "Journal Citation Report.”

$$
\text { http://sub3.webofknowledge.com/error/Error? }
$$

$$
\text { PathInfo=/\&Alias=WOK5\&Domain=.webofknowledge.com\&Src=IP\&Params=DestApp=U }
$$
A\&RouterURL=http://www.webofknowledge.com/\&Error=IPError.

Vasilevsky, N, T Johnson, K Corday, C Torniai, M Brush, E Segerdell, M Wilson, C Shaffer, D Robinson, and M Haendel. 2012. "Research Resources: Curating the New Eagle-i Discovery System.” Database : the Journal of Biological Databases and Curation 2012: bar067. doi:10.1093/database/bar067. http://www.ncbi.nlm.nih.gov/pubmed/22434835.

De Waard, Anita. 2010. "The Future of the Journal? Integrating Research Data with Scientific Discourse” (August 6). doi:10.1038/npre.2010.4742.1. http://precedings.nature.com/documents/4742/version/1.

Wahlsten, D. 1987. "Defects of the Fetal Forebrain in Mice with Hereditary Agenesis of the Corpus Callosum.” The Journal of Comparative Neurology 262 (2) (August 8): 227-41. doi:10.1002/cne.902620205. http://www.ncbi.nlm.nih.gov/pubmed/3624553.

Wiley Online Publishing. "Immunology Journal Instructions for Authors.” http://onlinelibrary.wiley.com/journal/10.1111/ (ISSN)1365-2567/homepage/ForAuthors.html.

_. 2013. "Journal of Comparative Neurology - JCN Antibody Database - Wiley Online Library.” http://onlinelibrary.wiley.com/journal/10.1002/ (ISSN)1096-9861/homepage/jcn_antibody_database.htm.

Yan, Yaohua, Xiang Tan, Xinmin Wu, Bai Shao, Xiaohong Wu, Jianhua Cao, Jian Xu, et al. 2013. "Involvement of Early Growth Response-2 (Egr-2) in Lipopolysaccharide-induced Neuroinflammation.” Journal of Molecular Histology 44 (3) (June): 249-57. doi:10.1007/s10735-013-9482-y. http://www.ncbi.nlm.nih.gov/pubmed/23307302.

Yook, Karen, Todd W Harris, Tamberlyn Bieri, Abigail Cabunoc, Juancarlos Chan, Wen J Chen, Paul Davis, et al. 2012. "WormBase 2012: More Genomes, More Data, New Website.” Nucleic Acids Research 40 (Database issue) (January): D735-41. doi:10.1093/nar/gkr954. http://www.pubmedcentral.nih.gov/articlerender.fcgi? artid $=3245152 \&$ tool $=$ pmcentrez\&rendertype $=$ abstract.

ZFIN. 2013. "ZFIN Zebrafish Nomenclature Guidelines - General Information - ZFIN Community Wiki." https://wiki.zfin.org/display/general/ZFIN+Zebrafish+Nomenclature+Guidelines. 


\section{Figure 1}

Resource identifiability across disciplines.

A. Summary of average fraction identified for each resource type. B-F. Identifiability of each resource type by discipline. The total number of resources for each type is: (B) antibodies, $n=703$; (C) cell lines, $n=104$; (D) constructs, $n=258$; (E) knockdown reagents, $n=210$; (F) organisms, $n=428$. The $\mathrm{x}$-axis is the average for each resource type across each domain. Variation from this average is shown by the bars, error bars indicate upper and lower $95 \%$ confidence intervals.

A

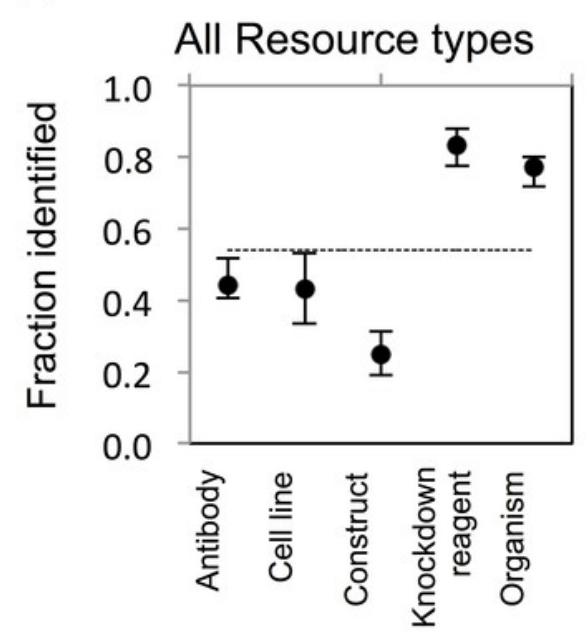

D

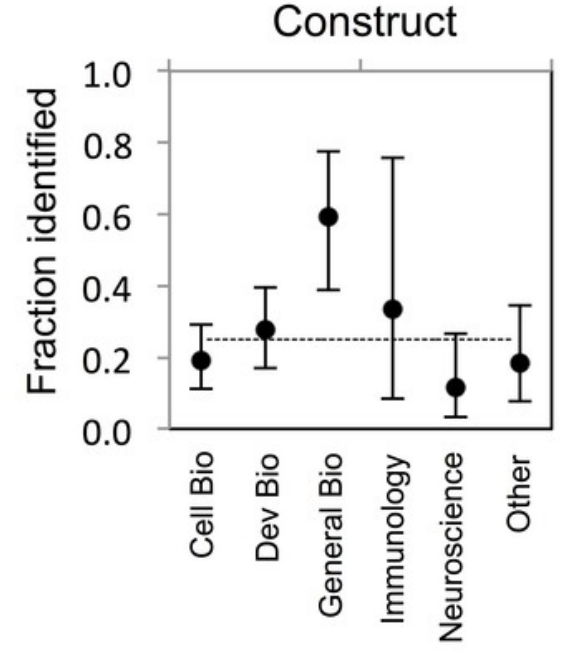

B

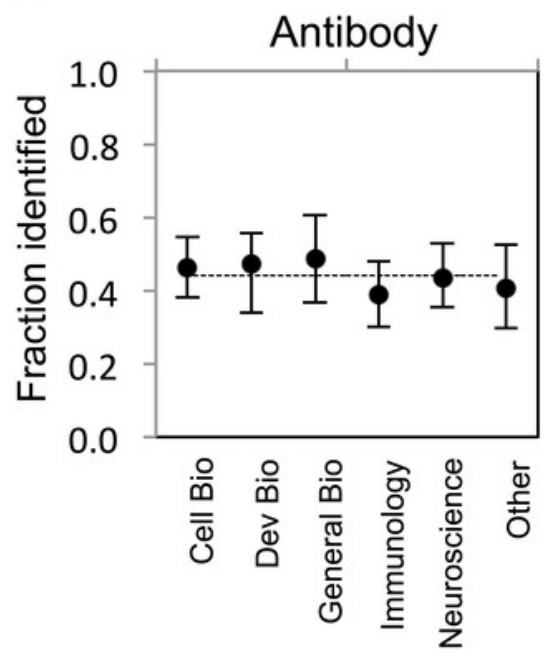

E



C

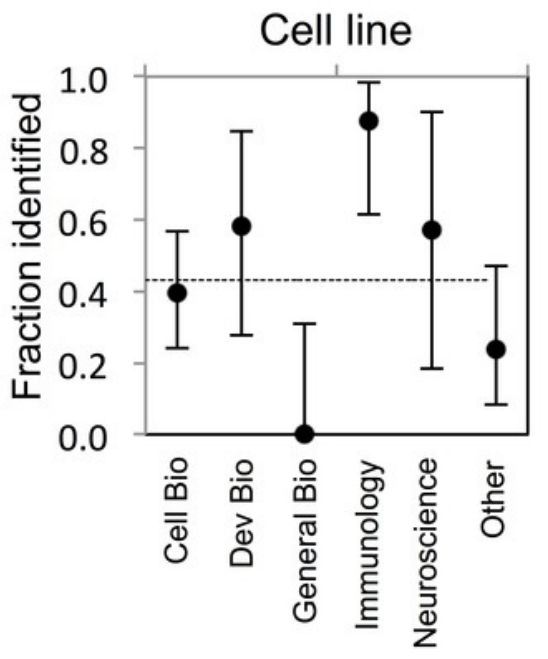

F

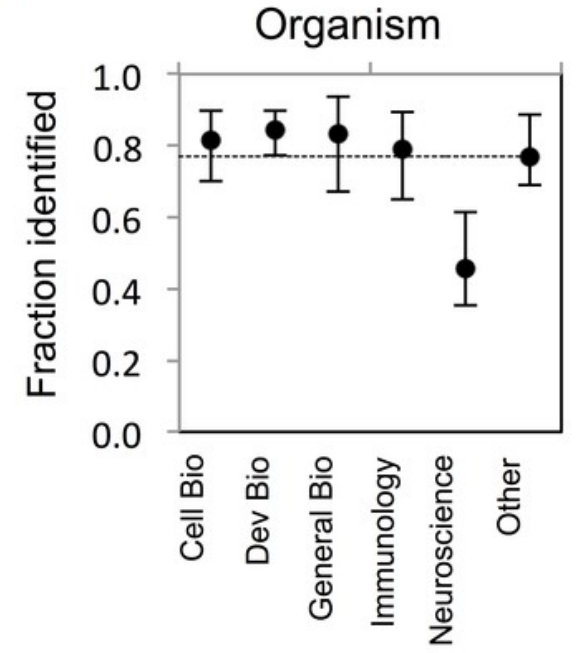




\section{Figure 2}

Resource identification rates across journals of varying impact factors.

A. An overview of fraction identified by impact factor for all resource types. B-F. Fraction identified by impact factor for each individual resource type. A higher number corresponds with a higher impact factor for each journal.

A

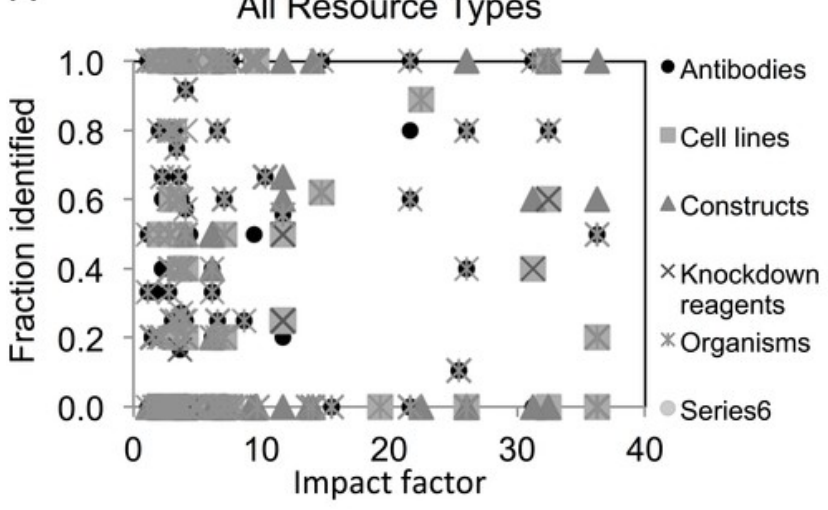

B

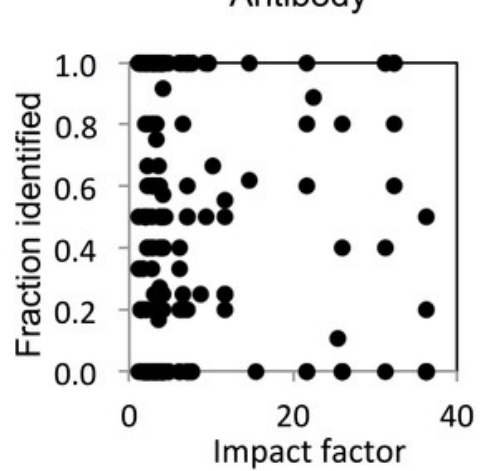

C

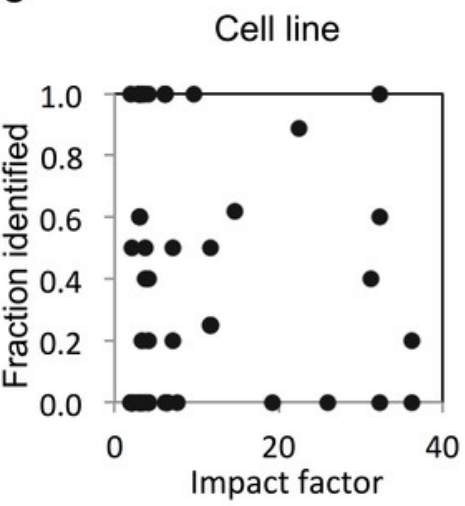

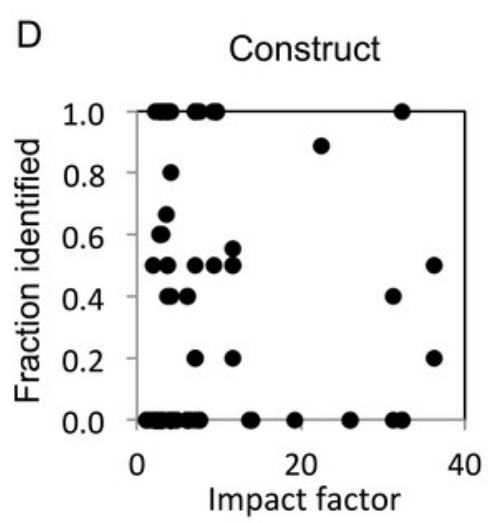



$\mathrm{F}$

Organism

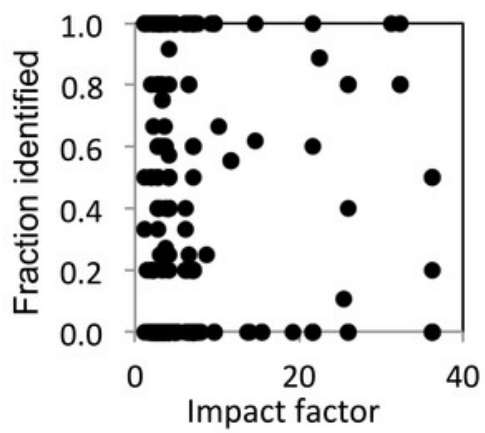




\section{Figure 3}

Identification of resource varies across journals with varying resource-reporting requirements.

The classifications of reporting requirements are summarized in the methods. A total of 53 out of 118 resources were identifiable in the stringent reporting guidelines category (17 papers were analyzed), 201 resources were identifiable out of 329 resources for the satisfactory category (48 papers were analyzed) and 662 out of 1,217 resources were identifiable in the loose category (182 papers were analyzed). Variation from this average is shown by the bars, error bars indicate upper and lower $95 \%$ confidence intervals.

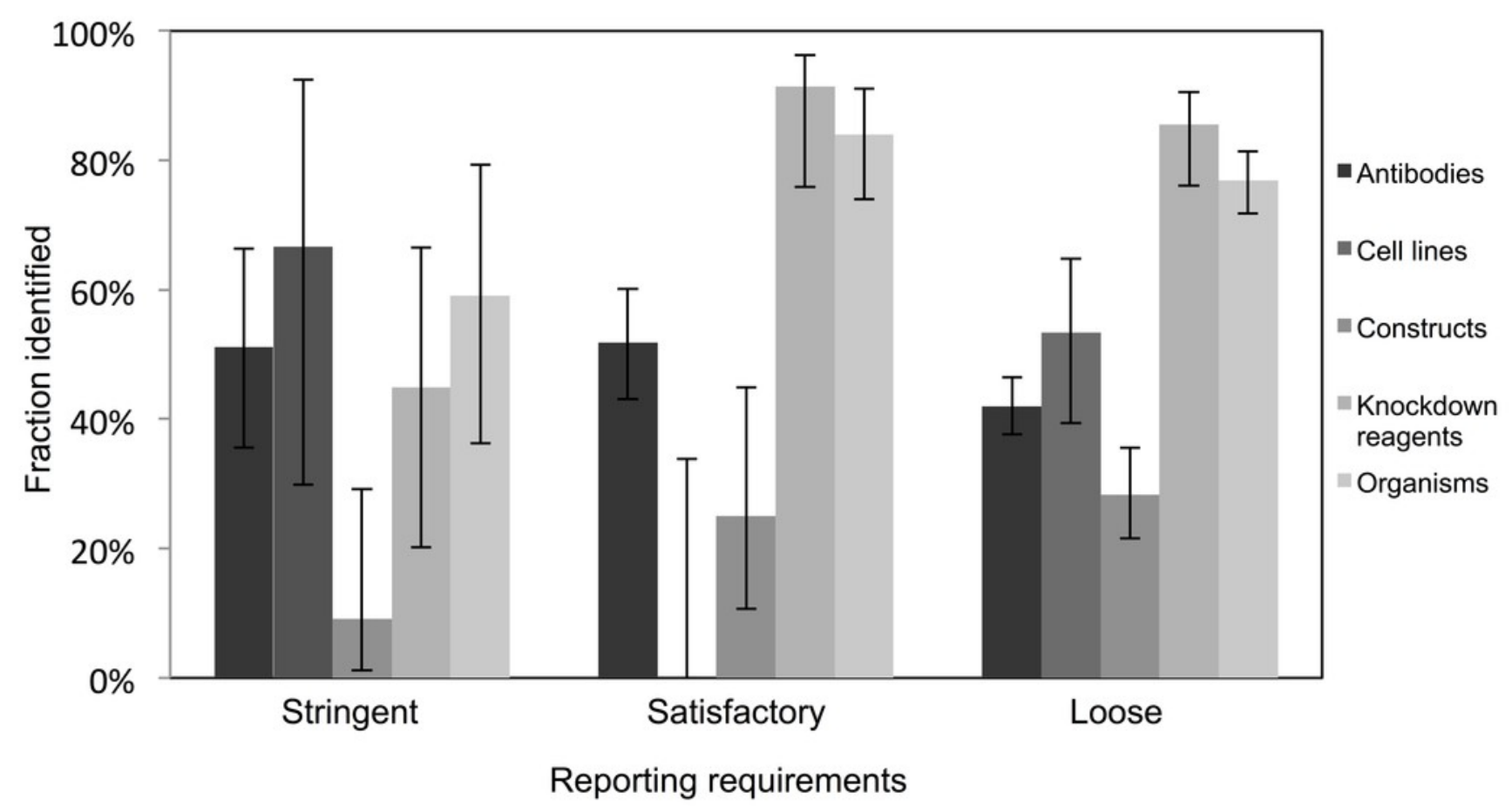




\section{Table $\mathbf{1}_{\text {(on next page) }}$}

Final numbers of identifiable resources in each domain. 


\section{Resourc} e type Antibod y Domain

Cell biology

General

biology

Immunolog

$y$

Neuroscien

ce

Other

Grand total

Cell

lines

Cell biology
Dev biology

Total number identifiabl e

68

312

Dev biology

General

biology

Immunolog

$y$

Neuroscien

ce

Other

Grand

total

Constru

cts

Cell biology

Dev biology

General

biology

Immunolog

$y$

Neuroscien

ce

Other

Grand total
69

68

36

48

60

31

Total number

of

resources papers

14

4

5

45

16

18

16

3

4

7

64
149

144

74

124

136

76

703

38

12

10

16

Total

number

of

34

44

19

28

41

24

190

17

5

5

6

6

9

48

17

19

8

3

7

12

38

258

66

Percen

tage

identifi

able

\section{$46 \%$}

$47 \%$

$49 \%$

$39 \%$

$44 \%$

$41 \%$

$44 \%$

$39 \%$

$58 \%$

$0 \%$

$88 \%$

$57 \%$

$24 \%$

Knockdo

wn

reagent

s

$\begin{array}{ll}\text { Cell biology } & 40 \\ \text { Dev biology } & 55 \\ \text { General } & 31\end{array}$

40

49

16

$82 \%$

76

22

$72 \%$

31

9

$100 \%$ 


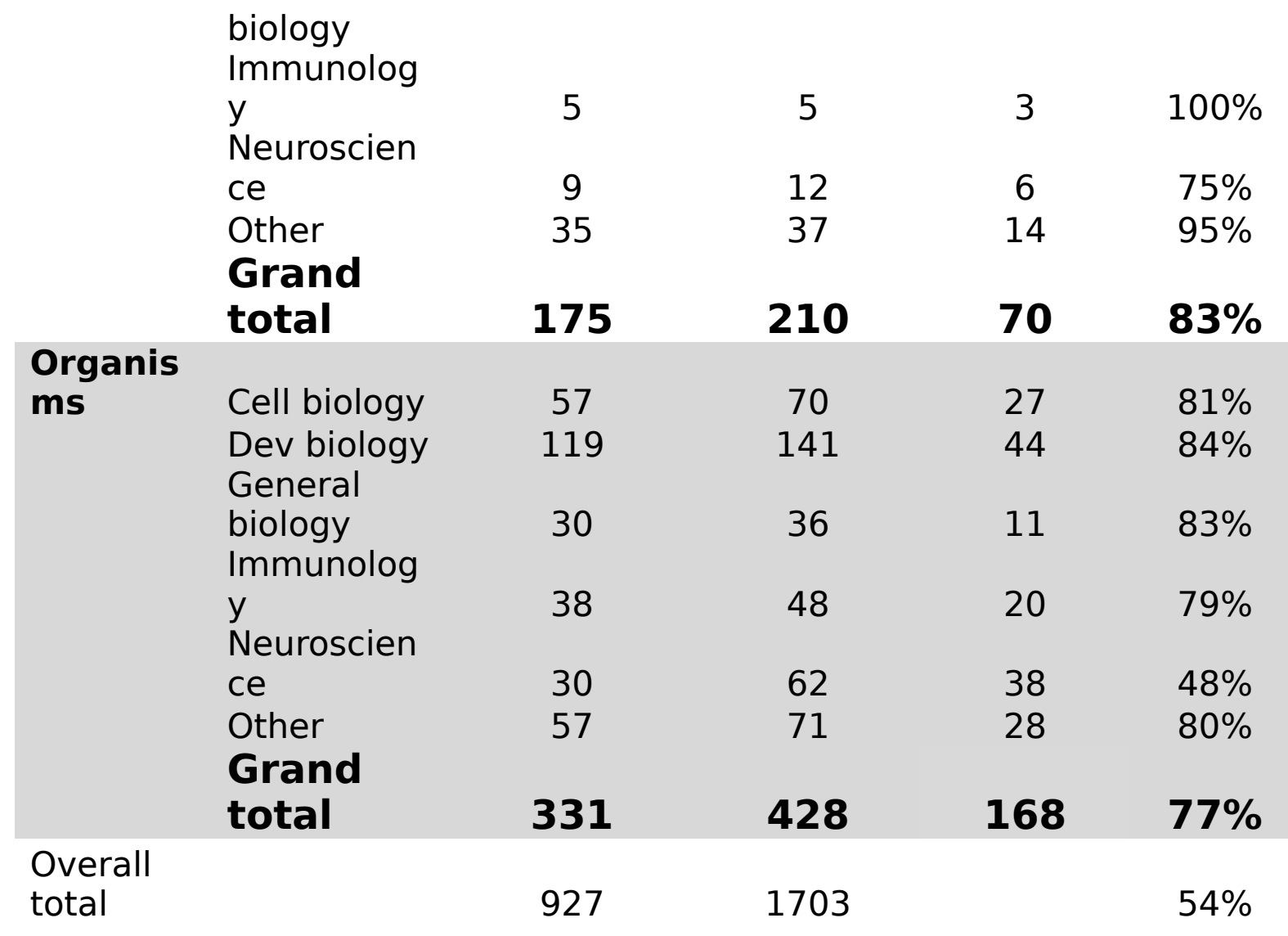

